\title{
NOTE \\ Successful Treatment of Disseminated Nocardiosis Complicated by Cerebral Abscess with Ceftriaxone and Amikacin: Case Report
}

\author{
F. Garlando, T. Bodmer, C. Lee, W. Zimmerli, \\ and M. Pirovino
}

\begin{abstract}
From the Department of Medicine, Kantonsspital Olten; the Institute of Medical Microbiology and Immunology, Kantonsspital St. Gallen; and the Division of Infectious Diseases, Department of Medicine,
\end{abstract} University Hospital, Basel, Switzerland

\begin{abstract}
We report the case of an 85-year-old female patient who suffered from disseminated Nocardia asteroides infection complicated by a cerebral abscess. Treatment with amikacin for 2 weeks and ceftriaxone for 6 weeks led to complete recovery, and there was no recurrence of disease over a follow-up period of 12 months after therapy. The use of ceftriaxone in combination with amikacin might significantly shorten the duration of treatment for patients with disseminated nocardiosis. This combination of antibiotics merits further investigation with use of a larger sample of patients.
\end{abstract}

Sulfonamides or trimethoprim-sulfamethoxazole is given as standard therapy for disseminated nocardiosis [1-3]. However, primary resistance to sulfonamides is well known $[1,4$, $5]$, late relapses are frequent $[4,6]$, and prolonged treatment is often required. Therefore, therapeutic alternatives are needed. In view of their good in vitro activity against species of Nocardia, ceftriaxone and cefuroxime seem especially promising $[5,7]$. Amikacin is another drug with excellent efficacy in vitro [5], in animal models [8], and in treatment of human disease $[4,9]$. Good clinical results have been observed with the synergistic combination of cefuroxime and amikacin [4].

We report the case of a patient with disseminated nocardiosis complicated by cerebral abscess who was successfully treated with ceftriaxone for 6 weeks and amikacin for 2 weeks.

An 85-year-old female patient was admitted to the hospital because of arthralgias and multiple skin lesions. On admission, the patient was afebrile; physical examination revealed multiple nodular reddish lesions on the left thigh and the lower abdomen that were painful and measured up to $1.5 \mathrm{~cm}$.

Laboratory analysis revealed mild leukocytosis (white blood cell count, $15.1 \times 10^{9} / \mathrm{L}$ ) with $40 \%$ neutrophils and a relative lymphopenia of $10 \%$. The Multi-Mérieux skin test (Institut Mérieux, Lyon, France) revealed complete anergy. Microscopic examination of pus obtained by puncture of one of the cutaneous lesions showed polymorphonuclear leukocytes without bacteria. Over the next 24 hours, further skin

\footnotetext{
Received 24 March 1992; revised 2 June 1992.

Reprints or correspondence: Dr. M. Pirovino, Medizinische Klinik, Kantonsspital, CH-4600 Olten, Switzerland.
}

Clinical Infectious Diseases 1992;15:1039-40

(c) 1992 by The University of Chicago. All rights reserved.

$1058-4838 / 92 / 1506-0015 \$ 02.00$ lesions appeared and signs of peritoneal irritation were observed. A repeated puncture of a cutaneous lesion revealed delicate, beaded, branching gram-positive filaments. Microscopic examination of the sputum showed the same microorganisms.

A presumptive clinical diagnosis of actinomycosis was made, and 20 million units of iv penicillin $G$ were administered daily. Because of generalized seizures that were assumed to be due to CNS toxicity associated with the use of penicillin, the treatment was changed to amoxicillin. On day 8 of treatment, a left-sided hemiparesis appeared. The computed tomography (CT) scan revealed a right-sided ring-enhancing hemispheric lesion suggestive of an abscess (figure 1). The lumbar puncture showed a normal cell count and a slightly elevated level of protein $(56.8 \mathrm{mg} / \mathrm{dL})$.

In the meantime, culture of a sample obtained by cutaneous aspiration revealed $N$. asteroides that was resistant to trimethoprim-sulfamethoxazole and produced penicillinase. Antibiotic treatment was changed to iv amikacin (MIC, 0.12 $\mu \mathrm{g} / \mathrm{mL}$ ) and iv ceftriaxone (MIC, $8 \mu \mathrm{g} / \mathrm{mL}$ ) (for microdilution method, see [10]). In vitro testing showed that the combination of ceftriaxone and amikacin did not have synergistic potential. During treatment with amikacin ( $200 \mathrm{mg}$ b.i.d. iv), a peak serum concentration of $13.6 \mathrm{mg} / \mathrm{L}$ and trough concentrations of $2.3-5.5 \mathrm{mg} / \mathrm{L}$ were measured.

Ceftriaxone was administered in a total daily dose of $4 \mathrm{~g}$ (divided in two doses). With this treatment, the patient's neurological deficits were markedly reduced; thus, therapy with amikacin was stopped after 14 days. Therapy with ceftriaxone was continued for 6 weeks. After 4 weeks of therapy, a second CT scan showed a marked regression of the lesion; a third CT scan of the brain 2 weeks after termination of treatment revealed no evidence of a persisting abscess. Clinically, the patient showed no residual neurological deficits, and the skin changes as well as the signs of peritoneal irritation had disappeared. 


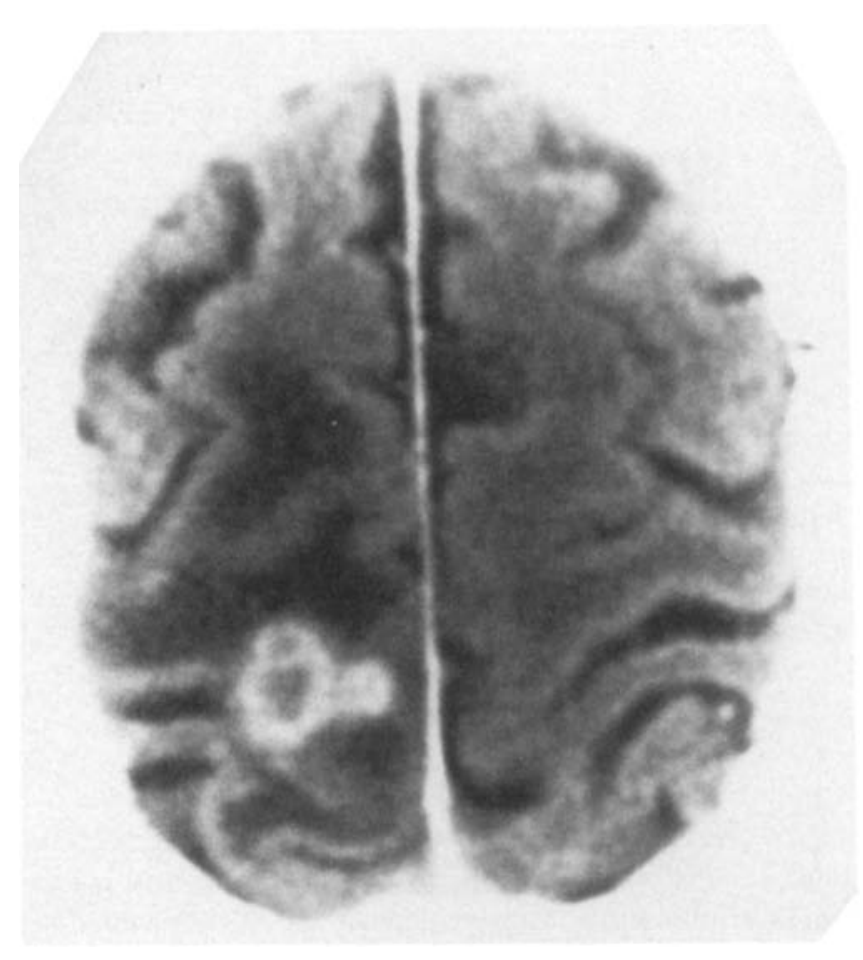

Figure 1. A CT scan taken at the beginning of antibiotic treatment revealing a right-sided parietal brain abscess with multilocular ring enhancement and edema.

Disseminated nocardiosis has a poor prognosis, with a mortality rate of $7 \%-44 \%[2,11,12]$. Treatment of nocardial brain abscesses with sulfonamides is usually recommended for up to 1 year [2]. Furthermore, since primary resistance and late relapses occur, alternative regimens are required. Among the various antibiotics tested, ceftriaxone, cefuroxime, and cefotaxime showed good in vitro activity against Nocardia [5]. Cefuroxime and amikacin have been shown to kill Nocardia synergistically in vitro [4], thus providing a rational basis for their clinical use.

In the murine model, cerebral nocardiosis responds better to amikacin than to trimethoprim-sulfamethoxazole [8]. However, these data should be interpreted with caution: concentrations of these drugs achieved in the CSF of humans are lower than those achieved in the brains of mice [13-15]; further problems result from the large variability of concentrations observed in the CSF of humans and the poor correlation between the concentrations measured in CSF and those in cerebral tissue $[14,15]$. Considering that the concentration of amikacin achieved in the CSF of humans is 10\%-15\% of that achieved in serum [12], a CNS effect can be anticipated with isolates of Nocardia that require a low MIC for inhibition; such an effect has been shown in our case, in which the MIC of amikacin was $0.12 \mu \mathrm{g} / \mathrm{mL}$ and serum concentrations of amikacin were between $2.3 \mathrm{mg} / \mathrm{L}$ and $13.6 \mathrm{mg} / \mathrm{L}$.
Our patient had an excellent response to therapy with ceftriaxone and amikacin. The neurological deficits disappeared after 3 weeks of treatment, a finding that correlated with the radiological evidence of regression after 4 weeks of treatment and the complete normalization of the computed tomogram 4 weeks later. On the basis of the low MIC $(0.12$ $\mu \mathrm{g} / \mathrm{mL}$ ) of amikacin and the relatively high MIC of ceftriaxone $(8 \mu \mathrm{g} / \mathrm{mL})$ in our case, we assume that amikacin had a major effect on the CNS manifestation. The possibility of synergism in vivo cannot be excluded despite its absence in vitro. The early institution of adequate antibiotic treatment may have been critical. The use of combination therapy with amikacin and ceftriaxone for patients with disseminated nocardiosis merits further investigation.

\section{References}

1. Wallace RJ Jr, Septimus EJ, Williams TW Jr, et al. Use of trimethoprim-sulfamethoxazole for treatment of infections due to Nocardia. Rev Infect Dis 1982;4:315-25.

2. Wilson JP, Turner HR, Kirchner KA, Chapman SW. Nocardial infection in renal transplant recipients. Medicine (Baltimore) 1989; 68:38-57.

3. Berkey P, Bodey GP. Nocardial infection in patients with neoplastic disease. Rev Infect Dis 1989;11:407-12.

4. Goldstein FW, Hautefort B, Acar JF. Amikacin-containing regimens for treatment of nocardiosis in immunocompromised patients. Eur $\mathrm{J}$ Clin Microbiol Infect Dis 1987;6: 198-200.

5. Dewsnup DH, Wright DN. In vitro susceptibility of Nocardia asteroides to 25 antimicrobial agents. Antimicrob Agents Chemother 1984; 25:165-7.

6. Stamm AM, McFall DW, Dismukes WE. Failure of sulfonamides and trimethoprim in the treatment of nocardiosis. Arch Intern Med $1983 ; 143: 383-5$.

7. Gombert ME. Susceptibility of Nocardia asteroides to various antibiotics, including newer $\beta$-lactams, trimethoprim-sulfamethoxazole, amikacin and $\mathrm{N}$-formimidoyl thienamycin. Antimicrob Agents Chemother 1982;21:1011-2.

8. Gombert ME, Aulicino TM, Du Bouchet L, Silverman GE, Sheinbaum WM. Therapy of experimental cerebral nocardiosis with imipenem, amikacin, trimethoprim-sulfamethoxazole, and minocycline. Antimicrob Agents Chemother 1986;30:270-3.

9. Meier B, Metzger U, Müller F, Siegenthaler W, Lüthy R. Successful treatment of a pancreatic Nocardia asteroides abscess with amikacin and surgical drainage. Antimicrob Agents Chemother 1986;29:1501.

10. Lorian V. Antibiotics in laboratory medicine. 2nd ed. Baltimore: Williams \& Wilkins, 1986.

11. Beaman BL, Burnside J, Edwards B, Causey W. Nocardial infections in the United States, 1972-1974. J Infect Dis 1976;134:286-9.

12. Palmer DL, Harvey RL, Wheeler JK. Diagnostic and therapeutic considerations in Nocardia asteroides infection. Medicine (Baltimore) 1974;53:391-401.

13. Yow MD. An overview of pediatric experience with amikacin. Am J Med 1977;62:954-8.

14. Richards ML, Prince RA, Kenulay KA, Johnson JA, Lefrock JL. Antimicrobial penetration into cerebrospinal fluid. Drug Intelligence and Clinical Pharmacy 1981;15:341-68.

15. Yogev R, Kolling WM. Intraventricular levels of amikacin after intravenous administration. Antimicrob Agents Chemother 1981;20:5836. 